\title{
How Many Cognitive Channels Should the Primary User Share?
}

\author{
Aaqib Patel, Member, IEEE, Md. Zafar Ali Khan, Senior Member, IEEE, S. N. Merchant, Member, IEEE, U. B. \\ Desai, Senior Member, IEEE, Lajos Hanzo, Fellow, IEEE
}

\begin{abstract}
Despite almost two decades of research, multiple challenges remain unresolved in Cognitive Radio (CR) scenarios, especially the fundamental problem of reliable spectrum sensing. Hence we propose a channel partitioning scheme (CPS) based CR relying on realistic imperfect spectrum sensing. The channels licensed to the primary user (PU) are partitioned into two sets. Channels in one of the sets are exclusively reserved for the PU, while those in the other can be accessed by both the PU and the secondary user (SU) after spectrum sensing. The PU prioritizes its transmissions over the two sets by first transmitting using the channels in the reserved set and then moves on to the unreserved set, if required. It is shown that this simple policy significantly improves the overall detection performance as well as the simultaneous achievable rates and reduces the overhead associated with sensing.
\end{abstract}

\section{INTRODUCTION}

Cognitive Radio (CR) was introduced to address the problem of spectrum imbalance where on one hand there are bands that are under-utilized, while others are crowded or are expected to become crowded in the near future [1]-[3]. CR allows that along with legacy transmissions of the primary user (PU), the secondary users (SU) are also allowed access to the under-utilized bands with appropriate safeguarding of legacy transmissions.

Since the term "Cognitive Radio" was coined back in the year 1999 by Mitola [1], tremendous interest has been shown by researchers from diverse backgrounds providing theoretical results, design guidelines and experimental confirmations of the CR benefits, highlighting both the technical and regulatory issues in allowing SU transmissions. The research has also attracted various standardization activities, as discussed in [4]. Yet, satisfactory solutions to many of the open problems still have to be found. Hence we shall enlist the major challenges that require attention, highlighting the fundamental hitherto unsolved fundamental problem of spectrum sensing.

Spectrum sensing is a key enabling technique of CR systems, since it provides the SU with the knowledge of the underutilized channels of the PU. Hence it has attracted substantial research efforts [5]. The fundamental issue in

Aaqib Patel, Md. Zafar Ali Khan and U. B. Desai are with the Department of Electrical Engineering, Indian Institute of Technology Hyderabad, Yeddumailaram, 502205 e-mail: aaqib,zafar,ubdesai@iith.ac.in.

S. N. Merchant is with the Department of Electrical Engineering, Indian Institute of Technology Bombay, Powai Mumbai, 400076, e-mail: merchant@ee.iitb.ac.in.

Lajos Hanzo is with the School of Electronics and Computer Science, University of Southampton, SO17 1BJ, email:lh@ecs.soton.ac.uk spectrum sensing is the lack of near-real-time, computationally efficient and reliable techniques. To fill this gap, we propose a novel scheme, which is simple yet effective in achieving the basic objectives of CR namely improving the overall spectral efficiency at low cost to the SU by improving the overall detection performance. The scheme requires limited cognition on behalf of the SU, while the PU is still oblivious to the SU transmissions.

We highlight the importance of spectrum sensing in detail next by discussing the various functions of CR that are directly dependent on the accurate, real time and practical spectrum sensing.

\section{IMPACT OF SPECTRUM SENSING}

Since CR networks have to perform intelligent tasks, there are multiple challenges that have to be addressed for the seamless operation of the SU along with the PU. We highlight some of these important challenges that are directly dependent on spectrum sensing and we also discuss the impact of inaccurate spectrum sensing on each of these challenges. We first note that any spectrum sensing technique has three qualities pertaining to its efficiency.

1) Accuracy - Accurate spectrum sensing is the key to safeguarding the PU transmissions, because inaccuracies result in interference between the PU and SU transmissions, which renders the communication unreliable.

2) Real-time - Near-real-time actions are required for providing the SU with sufficient time for communication and to prevent a state-change during sensing that could be caused by an excessive sensing duration.

3) Practical - Given that in general sensing is carried out at the SU transmitter, no a priori knowledge of the PU signal is available. At best some its statistical characteristics may be known.

We first summarize the impact of spectrum sensing in Fig. 1, where eight challenges have been highlighted that are directly dependent on spectrum sensing. Note that the accuracy of spectrum sensing is decided by the simultaneously achievable probabilities of missed detection and false alarm. For a given value of one these two parameters we wish to minimize the other. Unfortunately, this minimization comes at some computational complexity. Let us now discuss, how spectrum sensing affects each of these challenges.

- Self organizing and distributed decision making for cooperative spectrum sensing (CSS) - The basic components of CSS are constituted by the individual SUs that perform spectrum sensing at their locations. Any 


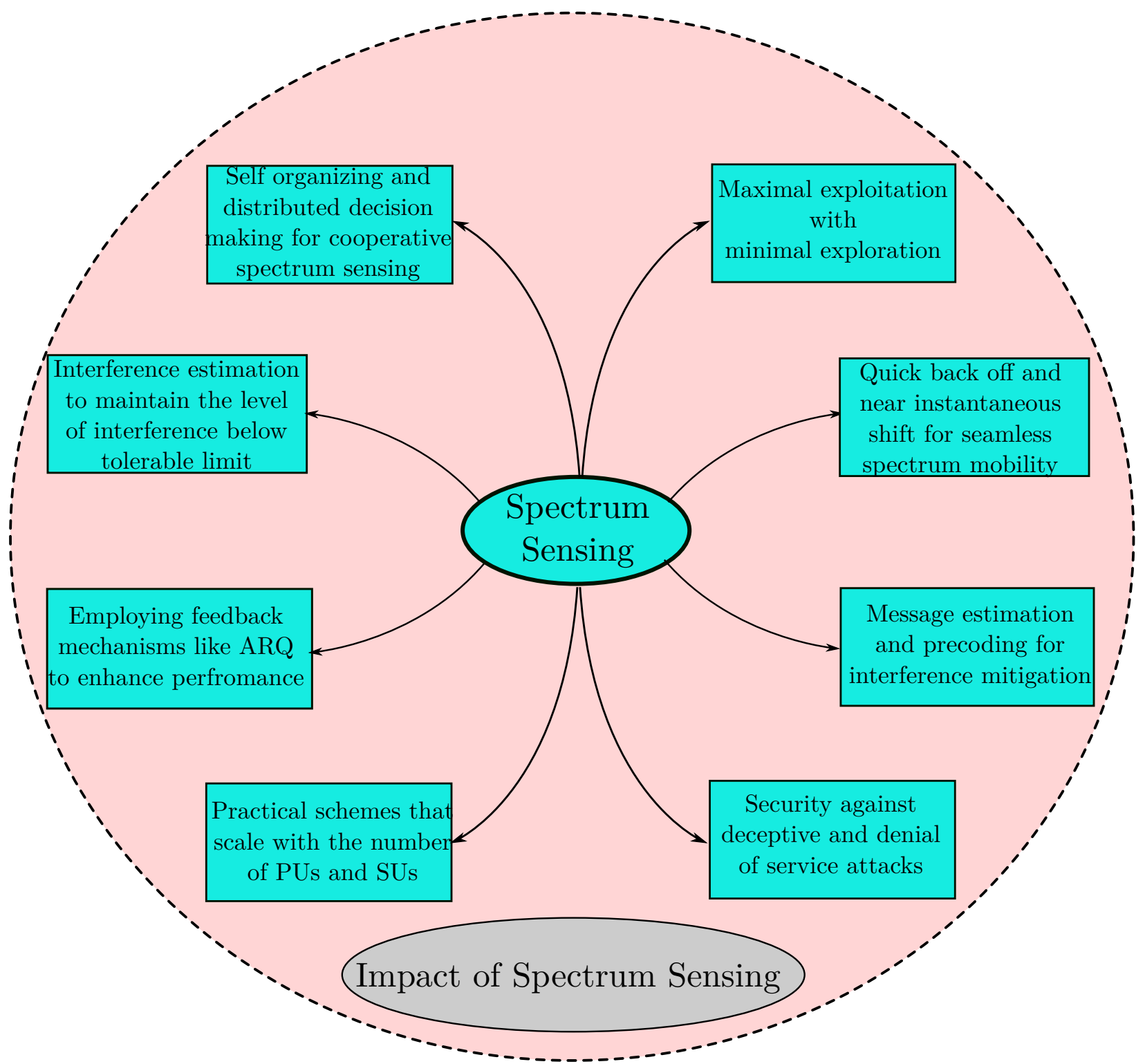

Fig. 1: The Impact of spectrum sensing on important CR functionalities.

inaccuracies in the spectrum sensing at each SU will have an impact at the central fusion node in taking the final decision by combining these individual decisions. Moreover, the choice of the spectrum sensing technique used will also have an impact on the cooperation models utilized by the network. Any computationally intensive technique will add to the overheads of the cooperating SUs. Moreover, it will be a challenge to acquire any knowledge of the incumbent for each SU without cooperating. As such this cooperation will again increase the overheads imposed [6]

- Spectrum exploitation vs. spectrum exploration Sensing sequentially over a wide and possibly discon- tiguous band of asymmetric channels has two aspects (i) cost of exploration and reward of exploitation. The cost of exploration per channel is increased when the spectrum sensing technique is computationally intensive. Moreover, sensing accuracy has a direct bearing both on the instantaneous and on the expected future rewards, hence ultimately on the exploitation. Minimizing the cost of exploration, while maximally exploiting the available channels [7] is the key to improving the overall spectral efficiency.

- Seamless spectrum mobility - The PU may have to use its spectrum at any instant or a mobile SU user may move in a zone, where the PU was already in 
communication on a channel the SU previously found free. Ensuring prompt SU-backoff, while supporting seamless spectrum mobility is thus very important for the SUs [8], so that the user experience is never unduly compromised. For effective implementation supporting continuous spectrum sensing is crucial and hence the time spent on sensing should be as low as possible.

- Interference estimation - To keep the interference imposed on the PU receiver below the tolerable level is the most important task, especially when multiple SUs transmit [9], so that the underlay CR mechanisms can be used to protect the Quality of Service (QoS) for the PU. The SUs have to perform spectrum sensing to limit the interference.

- Precoding for relaying and interference mitigation - Decoding of the PU message at the original source transmission rate by the SU to perform either realtime transmit precoding [10] or relaying [11] requires the knowledge of the instances when the PU transmits. Any inaccuracy would imply decoding noise and consequently the ensuing processes will be futile. Here accuracy of this process is crucial.

- Scalability - Accurate and practical spectrum sensing becomes important for networks supporting a high userdensity. Given the limited knowledge of other users the SUs have to perform accurate sensing. This challenge increases several folds, when cooperative spectrum sensing is imposed.

- Security - The PUs and SUs have to be protected from various types of attacks, such as PU emulation, denial of service and deceptive attacks [13]. The SUs may have to employ enhanced spectrum sensing techniques to differntiate between the PU and other SUs, when some SUs emulate the PU transmission characteristics. This adds a new dimension to spectrum sensing in the face of security threats.

- Utilizing Feedback- CR systems can benefit from error control mechanisms, such as ARQ [14]. If the PU receivers are close to the $\mathrm{SU}$ receivers, then it is more accurate and fruitful to perform spectrum sensing at the receivers. Using spectrum sensing at the receiver may also act as an aid to spectrum sensing at the transmitter and as such can result in more accurate sensing. However, this improvement will be challenged by a decoding delay. The impact of such a scheme on the performance attained has to be investigated. Surprisingly little attention has been paid to these systems compared to the aforementioned challenges.

Next we briefly study some of the existing spectrum sensing techniques in terms of the three requirements.

\section{A. Well known spectrum sensing techniques}

We emphasize that despite the enormous efforts invested in finding solutions to these problems, satisfactory spectrum sensing remain elusive. Signal detection theory (termed spectrum sensing in the context of CR) is a classic area of research, but the challenge is that any known detection technique satisfies only some of the triple requirements of accurate, practical and prompt reuse. Explicitly, energy detection is practical and prompt but not accurate enough, while cyclostationary detectors are practical and accurate but slow (since their complexity is huge) and finally matched-filter detectors are accurate and prompt but not practical (since full signal knowledge is assumed). These challenges call for innovative solutions. The impact of inaccuracy is felt most when an ongoing PU transmission is contaminated by interference due to missed detection and any detection technique having a low probability of missed detection $\left(p_{m d}\right)$ will inflict a less detrimental impact. In addition to missed detection another factor that significantly increases the number of collisions is the probability that a PU occupies a channel $p_{o}$. Hence the probability that there is a collision is simply $p_{o} p_{m d}$. Numerous techniques have been proposed for reducing the $p_{m d}$, while maintaining the same false alarm rate. We however, choose to concentrate on finding compelling techniques of reducing the impact of $p_{o}$. Hence we propose a channel partitioning scheme in the next section to achieve this goal. We explicitly state that we do not propose any new spectrum sensing technique, but reather we conceive a scheme that intelligently reduces the impact of PU transmissions that occur at random to reduce the probability that a collision occurs. However, before we discuss the solutions in detail, we define the notions of cooperation and cognition in a cognitive radio network, which will highlight the practicality of our approach.

\section{COOPERATION AND COGNITION}

Any CR network can be described using the following cooperation and cognition based classification:

- Cooperation offered by the PU network to the SU network or simply cooperation (this is not to be confused with cooperation amongst the SUs in cooperative spectrum sensing),

- Cognitive capability of the unlicensed user.

To safeguard the PU, some researchers propose that cooperation be offered by the PU in lieu of a suitable gain as exemplified by relaying gain - or by an improvement in overall spectral efficiency. However, there are others who are vehemently opposed to the idea of cooperation and wish to rely solely on the cognitive capabilities of the SU. In reality, it depends on the specific propagation and tele-traffic scenario, whether cooperation or cognition is more desirable. Striking a time-variant adaptive compromise between cooperation and cognition relying on

Fig. 2 is beneficial. A difference in any of the two will lead to a different scenario. This cooperation-cognition based classification will be useful for us to suggest practical models that are based on beneficial levels of cooperation and cognition. Let us now discuss some of the well-known cooperation and cognition scenarios. For ease of interpretation we have categorized both cooperation and cognition into three grades default, low and high. While default cooperation and cognition is the most basic form, which is assumed in typical models, low may be considered to be practical and high to be radical.

\section{- Level of Cooperation.}




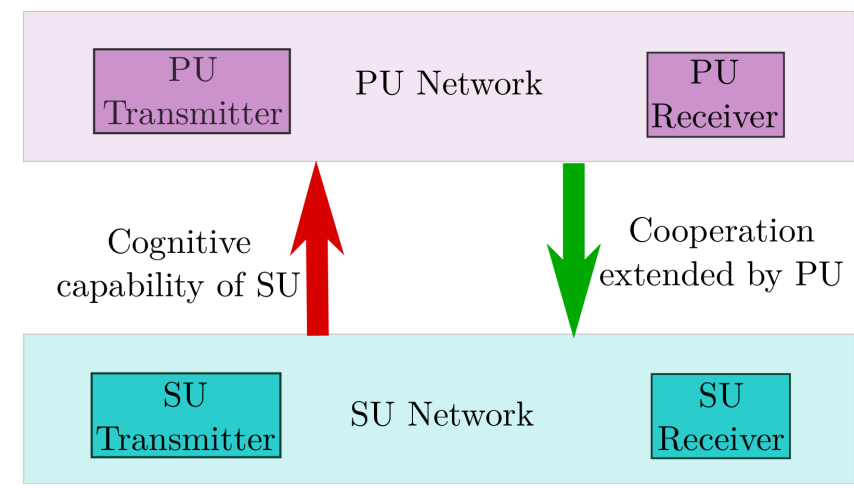

Fig. 2: A CR network characterized by the cooperation extended by the PU network and cognitive capability of the SU network

- Default level of cooperation: Unlike the command and control policy of the operation allocation schemes, the SU is allowed to access the licensed spectrum, whilst being oblivious to the PU .

- Low level of cooperation: The SU transmissions are allowed in the licensed band and the PU can tolerate a certain level of the SU interference. A plethora of different SU impositions may occur, such as (i) instantaneous interference below a predefined threshold, (ii) rate limitations, whilst a minimum transmission rate has to be maintained for the PU, (iii) outage constraints, so that the SU transmissions impose only a limited outage probability on the PU and (iv) probability of collision constraints, which requires that the SU has to employ a sensitive PU detector to avoid collisions. Naturally, many other constraints may be imposed, but the ones mentioned above encompass the majority of ideas suggested in the literature.

- High level of cooperation: The PU cooperates extensively with the SU. It can do so by precisely planning its transmission opportunities and informing the SU about it. For example, in such scenarios the SU may compete for offering relaying services to the PU in exchange for superimposing their payload on that of the SU.

\section{- Level of Cognition.}

- Default level of cognition: The simplest action for the SU is to prepare for the worst-case scenario, when a PU is always transmitting. Although this is not a typical scenario in a cognitive radio, it hinges on the fact that there are no fail-safe methods of identifying the absence of the PU transmission. Any strategy that deviates from the worst-case scenario will impose some interference on the PU network.

- Low level of cognition: The SU can perform spectrum sensing in order to detect the PU's presence with some non-zero probability of error over a limited range of frequencies. This works only when the PU is ready to tolerate some non-zero probability of collision (low level of cooperation offered by PU).

- High level of cognition: The SU can perform perfect spectrum sensing. Furthermore, it may also be able to estimate the PU's transmission to its receivers. This will allow the SU to transmit freely, when the PU is absent and to perform transmit precoding, when the PU is present (depending upon the PU's level of cooperation).

The challenge is to identify the most appropriate combination of cooperation and cognition. The basic requirement is that the CR scheme has to be practically feasible and simultaneously be spectral-efficient. In the presence of the basic default cooperation, perfectly safeguarding the PU will require high level of cognition, while in the presence of default cognition a high level of cooperation is required from the PU for achieving improved spectral efficiency. However, neither a high level of cooperation nor a high level of cognition are practically feasible. Having a low level of cooperation combined with default cognition or vice versa will be spectrally inefficient. We are then left only with schemes that operate at low level of cooperation and cognition. The challenge is to exploit this limited level of cooperation and cognition at hand and to create a CR system having a high spectral efficiency.

In the context of low-level cooperation and cognition we first propose a simple channel partitioning scheme (CPS), which is described next. It will be shown subsequently that this partitioning helps us improve accuracy as well reduce the overall complexity associated with a particular spectrum sensing technique, even when using the low-complexity energy detector.

\section{Channel Partition Scheme}

The uncertainty in the PU's transmission instants coupled with the imperfect spectrum sensing of the SU increases the chances of collisions between the PUs and SUs. However, upon appropriately partitioning the channels into two sets, one of the sets may be exclusively reserved for the PU transmissions, while the other set allows the SU and PU to coexists 1,2 . The packets in the PUs buffer arrive according to some wellbehaved (finite mean and variance) discrete distribution. If the number of packets that arrive in a unit time interval is lower than that of the reserved channels the PU selects the required number of channels uniformly at random in the reserved set. By contrast, if the number of packets is higher than that of the reserved channels, then the PU occupies all the channels in the reserved set and picks the remaining channels uniformly at random from the unreserved set. In short, the PU first transmits in its reserved set and when required, it moves on

\footnotetext{
${ }^{1}$ We do not assume that the PU has to reserve a contiguous band of channels. In fact the PU may reserve the channels best suited for its communication. The SUs are assumed to take what they wish.

${ }^{2}$ The PU may change the reserved set after some duration, which is assumed to be long enough so that communicating it to the SU does not affect the rate calculations.
} 


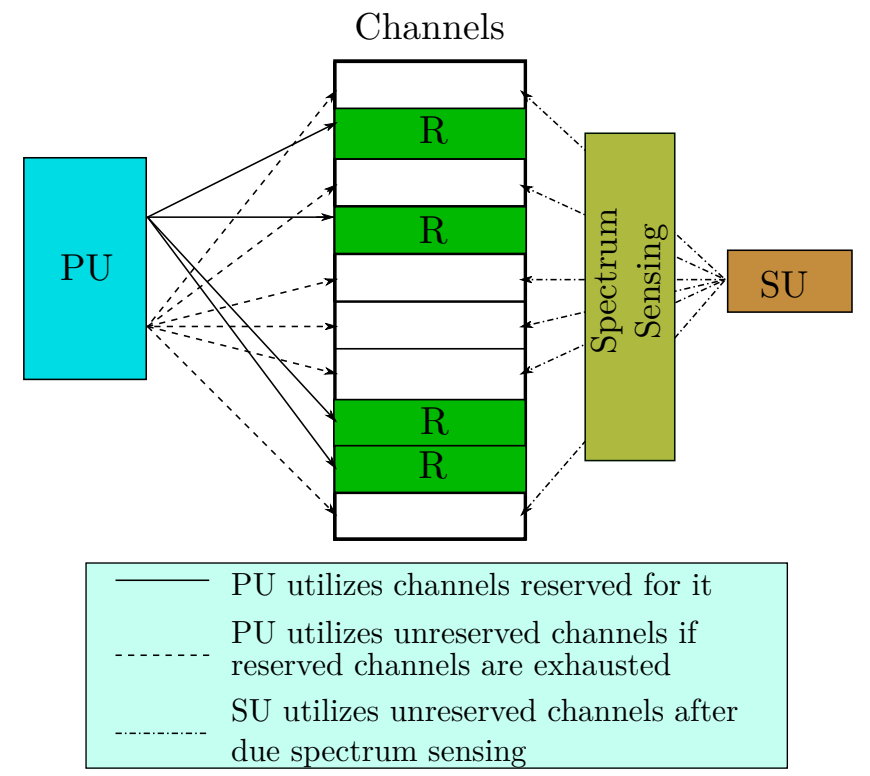

Fig. 3: The channel partitioning scheme illustrated.

to the unreserved set. The SU on the other hand senses only the channels in the unreserved set and transmits accordingly. The scheme is represented diagrammatically in Fig. 3. In our comparisons we shall term the scheme with no reservations as the traditional scheme (TS). ${ }^{3}$ Note that only a modest complexity is imposed by first transmitting in a particular set of channels in the context of CPS, which allows us to support the following two key properties:

- In the reserved band, CPS completely safeguards the PUs from the SUs.

- In the unreserved band - Compared to the traditional scheme, CPS reduces the probability of collision, since the uncertainty associated with the PU transmission is reduced.

To implement CPS, we have to answer the following questions:

- How many channels to reserve for CPS? Less reservations lead to the TS, while more reservations lead to the non-cognitive scenario.

- Which of the channels to reserve for CPS?

If the performance criterion is averaged over a long term, then we can safely assume that all the channels have the same performance. In this case only the first question of calculating the optimal number of channels to be reserved remains. But even this problem is hard in general. However, under some practical assumptions we can infer explicit rules for the optimal number of channels to be reserved. Nevertheless, it is always possible to plot the number of channels to be reserved against the probability of channel occupancy for the PU.

\footnotetext{
${ }^{3}$ Similar ideas for reservations have been suggested in the time domain in [15] as packet reservation multi-access. Complete sharing, complete partitioning and virtual partitioning have also been suggested for multi-access [16].
}

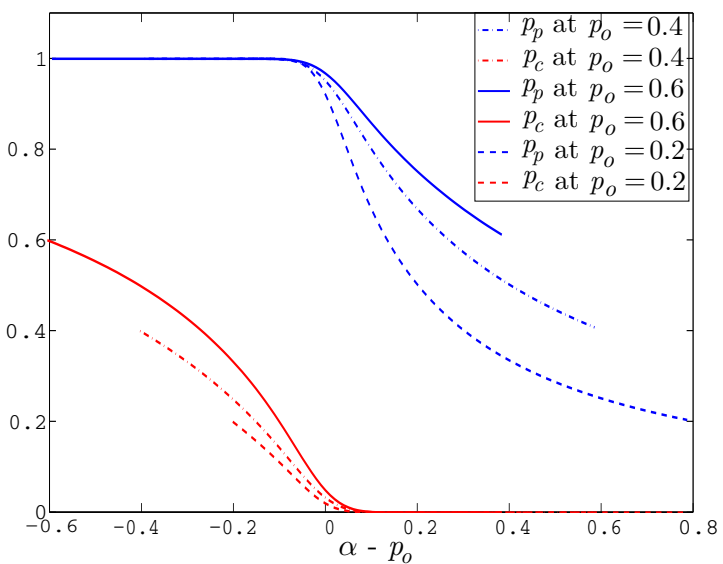

Fig. 4: Occupancy in the reserved set of channels $p_{p}$ and in the unreserved set of channels $p_{c}$.

\section{A. Performance Improvement}

Let us now study the performance improvement achieved using CPS. The parameters of interest that will be used for quantifying the performance improvements attained are defined in Table I. To better understand the performance improvement attained, we note that the uncertainty in the PU transmission is reduced due to CPS. This is because the reserved band is now heavily occupied, while the unreserved band is sparsely occupied. Fig. 4 plots the probabilities of occupancy in the reserved and the unreserved set. We observe that if the number of channels is carefully chosen, then $p_{p}$ is close to unity, whereas $p_{c}$ is close to zero. We show two specific facets, where the performance is improved, as follows

- Average detection performance: CPS reduces the number of collisions by reducing the occupancy in the unreserved band, while simultaneously protecting the PU from the SU in the reserved band. In essense the probability of missed detection is reduced. It will then be interesting to see, what is the cost incurred in terms of the probability of false alarm. We observe that the CPS results in an equivalent (imaginary) detector and we demonstrate that this equivalent detector is superior to the detector used in the TS. We do not claim the conception of a new detector, but nonetheless we improve the detection performance. To compare the detection performance of the CPS and TS, we employ an energy detector in both the schemes, where the sensing in the CPS is being restricted to the unreserved channels. Fig. 5a plots the Receiver Operating Charecteristic (ROC) curve for energy detection both for the traditional scheme and for the CPS. To elaborate, the ROC represents the minimum values of $p_{f a}$ achievable for a given $p_{m d}$ and vice versa. The curve for CPS is obtained by exploiting the following two relationships that compare the probabilities of missed detection $\left(p_{\hat{m} d}\right)$ and false alarms $\left(p_{\hat{f} a}\right)$ that occur in CPS to that of the TS. We note that $\hat{p}_{m d}$ is the 
TABLE I: Description of some variables

\begin{tabular}{|l|l|}
\hline Variable & Description \\
\hline$p_{o}$ & Probability that PU occupies a channel without reservation \\
\hline$p_{m d}$ & Probability that the SU fails to detect an ongoing PU transmission \\
\hline$p_{f a}$ & Probability that the SU fails to detect a transmission opportunities \\
\hline$p_{p}$ & Probability that the PU occupies a channel in the reserved set \\
\hline$p_{c}$ & Probability that the PU occupies a channel in the unreserved set \\
\hline$P_{p}, P_{c}$ & The average power available per channel with the PU and the SU respectively \\
\hline$R_{p}, R_{s}$ & The simultaneous data rates achievable for the PU and SU respectively \\
\hline$a, b$ & The cross link gain from the PU to the SU and the SU to the PU respectively \\
\hline$N$ & Number of channels with the PU \\
\hline$\alpha$ & Fraction of channels to be reserved for PU \\
\hline
\end{tabular}

ratio of the average number of collisions in the system to that of the average number of PU transmissions while $\hat{p}_{f a}$ is the ratio of the average number of transmission opportunities missed by the SU to the number of transmission opportunities that were presented by the PU. Consequently, it can be shown that,

$$
\begin{aligned}
& \hat{p}_{m d}=p_{m d}\left(1-\frac{p_{p}}{p_{o}} \alpha\right), \\
& \hat{p}_{f a}=p_{f a}+\frac{\alpha\left(1-p_{p}\right)\left(1-p_{f a}\right)}{1-p_{o}} .
\end{aligned}
$$

Note that in the above relationships, $p_{p}$ varies with the number of channels reserved $(\alpha N)$. The more channels are reserved for the PU, the lower will be the value of $p_{p}$.

Next, we discuss the procedure employed for plotting the ROC using these relationships. In TS, for a given $p_{m d}$, the best the energy detector can offer is $p_{f a}$, while the other detector parameters remain fixed. For a pair $\left(p_{f a}, p_{m d}\right)$, we vary the fraction $\alpha$ of channels reserved for the $\mathrm{PU}$, which results in a range of values of $\hat{p}_{m d}$ and $\hat{p}_{f a}$. Plotting $\hat{p}_{f a}$ versus $\hat{p}_{m d}$ gives us one curve. We obtain a family of such curves, one for each such pair $\left(p_{f a}, p_{m d}\right)$. The closure of the family (set) of curves gives the equivalent ROC curve for CPS, namely for a given $\hat{p}_{m d}$ the curve that contains infimum over all possible values of $\hat{p}_{f a}$ offered by the family. The performance improvement is substantial as seen in Fig. 5a. The CPS thus provides energy detection along with the much needed third attribute of accuracy, given that it is both prompt and practical.

The performance improvement attained can be explained as follows. Let us consider an ED operating at $\left(p_{m d}, p_{f a}\right)$. If there is no reservation, i.e. $\alpha=0$ then $\hat{p}_{m d}=p_{m d}$ and $\hat{p}_{f a}=p_{f a}$. Now we observe from Fig. 4 that as $\alpha$ increases, the value of $p_{p}$ remains nearconstant and equal to unity for a significant range of the values of $\alpha$. This implies that for all such values of $\alpha$, we can modify (1) to get $\hat{p}_{m d}=p_{m d}\left(1-\frac{\alpha}{p_{o}}\right)$ while $\hat{p}_{f a}=p_{f a}$. Hence for a given probability of false alarm, CPS achieves better performance in terms of its probability of missed detection than TS. And since this is true for all $\left(p_{m d}, p_{f a}\right)$ that are possible for the given $\mathrm{ED}$, the ROC of the equivalent (imaginary) detector is superior to that of the TS.

In simpler terms, for an appropriate number of reserved channels, the average number of opportunities missed by the SU is nearly the same for the CPS and TS, while the average number of collisions in CPS is substantially lower in CPS than in TS.

- Simultaneous ergodic achievable rates: A direct consequence of the improved detection is the improvement in the achievable rates. Fig. 5b plots the rates of the PU and SU that can be simultaneously attained (the achievable region). The achievable rate region of the CPS shows a substantial improvement over that of the traditional scheme. In particular, the PU gets near-ideal capacity for substantial non-zero SU rate, unlike the traditional scenario.

\section{B. Optimal fraction of channels to reserve for the $P U$}

Next we answer the interesting question of what is the optimal fraction of channels the PU should reserve for ensuring that the sum-rate of the PU and SU is maximized? This could also be modified to ask, what is the minimum number of channels to be reserved for the PU in order to attain its required rate (with an infinitesimally small tolerance). Under our assumptions, an explicit expression can be derived that is asymptotically optimal, when the number of channels tends to infinity, which is as follows

$$
\alpha^{*}=\frac{\left\lfloor\sqrt{N p_{o}\left(1-p_{o}\right)} Q^{-1}\left(\frac{A}{A+B}\right)+N p_{o}\right\rceil}{N} .
$$

where $A$ is the average rate that the SU achieves due to correct detection and $B$ is the reduction in rate due to missed detection $^{4}$. This equation is valid under the assumption that

\footnotetext{
${ }^{4}$ The exact expressions of $A$ and $B$ will change, depending on the specific signalling and modulation schemes. For our plots that follow we have assumed having ideal capacity achieving Gaussian signalling. The results should not differ in nature.
} 


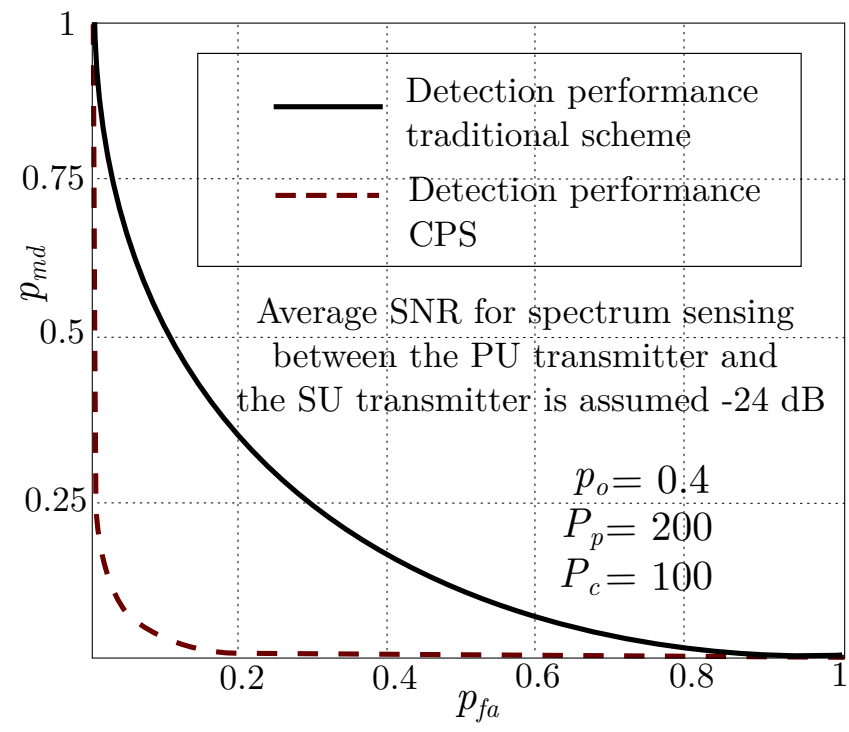

(a) ROC curves of both the traditional scheme and of the CPS using ED.

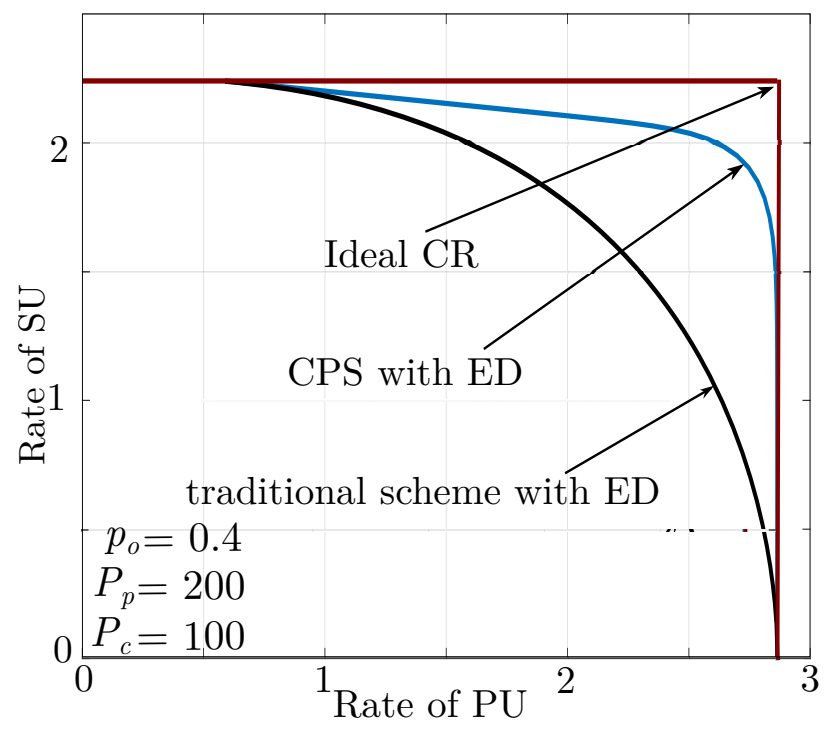

(b) The achievable rate region with energy detection of both the traditional scheme and of the CPS.

Fig. 5: Superior performance of CPS over TS.

the PU packets arrive according to a binomial process with $N$ trials having a packet arrival probability $p$.

The procedure of obtaining the expression for $\alpha^{*}$ is described next. Observe that defining $\alpha N$ as the number of channels reserved makes $\alpha$ a discrete variable. A standard method routinely employed for optimizing a discrete variable is to formulate a difference equation, which is anologous to the method of setting the derivative of a continuous function equal to zero. The difference equation is obtained by equating the difference in the objective function at two consequtive values of the discrete variable to zero. In our scenario the objective function is the sum of the rates of the PU and the SU, which is denoted by $C_{\text {sum }}(\alpha)$ for a given $\alpha$. The difference equation is then $C_{\text {sum }}\left(\alpha+\frac{1}{N}\right)-C_{\text {sum }}(\alpha)=0$.

The solution of this difference equation is $\alpha^{*}$. The simplified difference takes the form of an equation in which the left hand expression is a complementary binomial distribution function having a mean of $N p_{o}$ and a variance of $N p_{o}\left(1-p_{o}\right)$ and the right hand expression is $\frac{A}{A+B}$ that is seen in the expression of $\alpha^{*}$ above. Finally, to obtain a closed form expression for $\alpha^{*}$, we employ the Gaussian Approximation for the binomial distribution of the mean $N p_{o}$ and of the variance $N p_{o}\left(1-p_{o}\right)$. The employment of the Gaussian approximation gives us the $Q$-function, which we invert. Finally, since the number of channels reserved is an integer, we round off the continous value obtained upon inversion (denoted by $[.7$ ) to get our result. An explicit expression provides the PU with a rule that is universal.

For a set of parameters we also compute this value using a computer program. Fig. 6 plots the optimal fraction of channels $\alpha^{*}$ to be reserved against the probability $p_{o}$ that the channel is occupied by the PU. The curve is staircase-shaped, which is expected, since the number $\alpha^{*} N$ must be an integer. Moreover, $\alpha^{*}$ increases with $p_{o}$, closely following a value slightly less than $p_{o}$, which indicates that the PU should typically reserve slightly less channels than the average number of channels it occupies. Note that both the achievable rate region improvement and the optimal fraction of channels are dependent on the system parameters. In this article the assumptions are restrictive for the SU. However, any assumption that relaxes the constraints for the SU, while safeguarding the PU will improve the achievable rate region. For example, if the $\mathrm{SU}$ is not required to meet a per channel constraint of $P_{c}$ and it is instead allowed to distribute its total power over the number of active channels, we can improve the SU's achievable rates and consequently the value of $\alpha^{*}$ will decrease, while the PU will continue to achieve the same rate. If the receivers are able to decode the messages from other transmitters, then again, the value of $\alpha^{*}$ changes in favour of the user that is able to decode and cancel the maximum amount of interference. In such a case the SU will be able to transmit at significantly higher rates, provided that both receivers can cancel interference. An essential feature of CPS is that it is able to adjust to the amount of interference by suitably choosing a different $\alpha^{*}$ in favour of the user, whose achievable rates increase (as a result of some change in parameters). Hence, even with an increase in the interference power, the achievable rates in CPS remain more or less the same. This cannot happen in the traditional scenario.

Similarly, one can ask the minimum number of channels required for providing the $\mathrm{PU}$ with a minimum rate of $\bar{R}_{p}$. The optimal value of $\alpha$ is the solution to the equation $\left(1-\bar{\alpha}^{*} p_{c}=\right.$ 


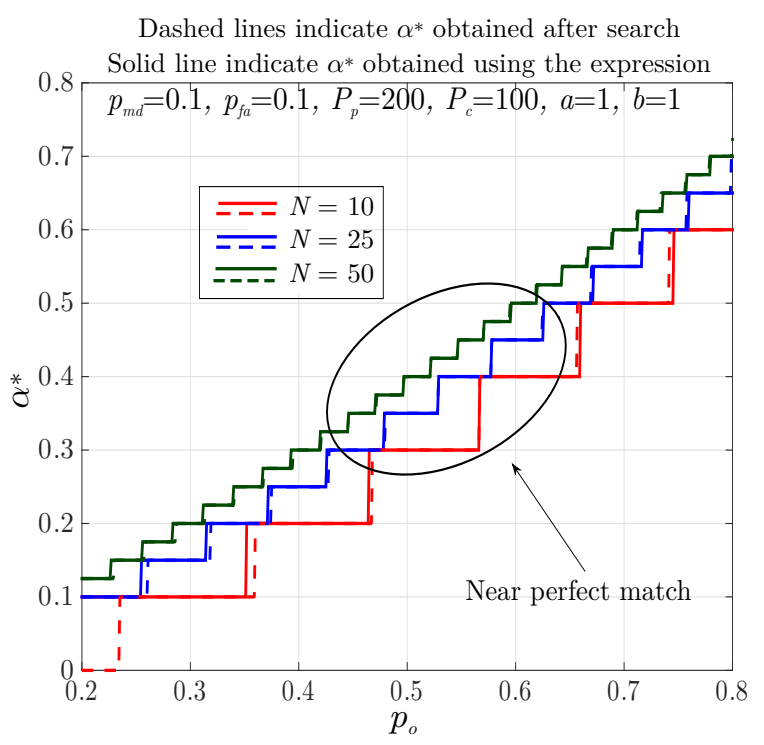

Fig. 6: The optimal fraction of channels to be reserved both by computing using a program and also plotting the theoretical expression

$C)$, where $C=\frac{p_{o} \bar{A}-\bar{R}_{p}}{\bar{A}-\bar{B}}$. Here $\bar{A}$ and $\bar{B}$ are full single user PU rates obtained with the aid of complete reservation and with no reservation, respectively. This expression is obtained by solving the expression of the PU rate $R_{p}=\bar{R}_{p}$ and exploiting the fact that $\alpha p_{p}+(1-\alpha) p_{c}=p_{o}$. Observe that $p_{c}$ is also a decreasing function of $\alpha$ and hence the higher the value of $R_{p}$ the lower the value of $\bar{\alpha}^{*}$. becomes

The selection of the optimal number of reserved channels in terms of some optimality criterion is also useful for incorporating scaling. As the number of PUs increase, they may want to reserve different channels for themselves. As such a particular reservation will be arranged either centrally or in a distributed manner, which can optimizes the performance attained. The performance criterion, for example, can be a mimimum rate-guarentee for each $\mathrm{PU}$ or the maximization of the sum rate of the PUs only. As such, the CPS will be useful under any circumstances in terms of reducing collisions. It is non-trivial though to comment on optimal reservations in terms of any arbitrary performance criterion. Clearly, this would require further study.

\section{Reduction in sensing overhead}

Spectrum sensing constitutes an overhead both in terms of energy and time spent. By restricting the spectrum sensing action to the unreserved band of CPS eliminates unnecessary spectrum sensing, which would have not lead to a transmission opportunity. In TS the specific fraction of spectrum sensing attempts that would lead to a transmission opportunity remains limited due to PU occupancy. By contrast, in the unreserved band most attempts result in desired communication. Further- more, since only a fraction of channels is tested, the computational complexity associated with sensing is also reduced.

\section{An example}

We now demonstrate the effectiveness of our scheme using a simple example that consists of $N=50$ channels. The probabilities of missed detection and false alarm for some detector is assumed as $p_{m d}=0.2$ and $p_{f a}=0.4$, while the link gains including the cross link gains are kept equal to unity. The average transmit powers $P_{p}=200$ and $P_{c}=100$. The table clearly demonstrates that the detection performance is improved substantially, because $\hat{p}_{m d}$ reduces to below 0.1 , whereas $\hat{p}_{f a}$ hovers around 0,4 . Also, the sum rates are always greater in CPS when $\alpha^{*}$ fraction of channels are utilized.

\section{DISCUSSIONS AND CONCLUSIONS}

A natural question arising is, how well CPS scales with the traffic intensity. Since we have considered a generic packet arrival model for the PU, the analysis remains valid, regardless of how many PUs are present. On the other hand, the resource allocation amongst the SUs hinges on the standard scheduling problem, which has been extensively studied. Our analysis can be readily extended to underlay-based CRs by also allowing the SU transmit at low rates or low power, so that the PU QoS is maintained in the reserved band. However, such a solution may either become spectrally inefficinent if the SUs interference is limited or energy-inefficient, if the SUs' transmit rate is limited. We can also arrange for more sophisticated PUcooperation in order to transmit on channels according to a specific priority order. Consequently the SUs will transmit in a reversed order to minimize the impact. But we note that this solution is not of generic nature and it does not scale with the number of PUs and SUs.

In conclusion, the fundamental limitations of spectrum sensing were investigated by conceiving the CPS philosophy advocated. It was shown to significantly improve both the detection performance and the resultant achievable rates. The reduction in the overhead of spectrum sensing was shown as an added advantage, since CPS used a prompt and practical energy detection based sensing technique and intelligently improved the accuracy. A key feature of the CPS is that it reduces the uncertainty associated with the PU transmissions and provides the SU with ample opportunities for transmission. The CPS was also shown to be robust to interference fluctuations, which was achieved by suitably modifying the fraction of reserved channels.

\section{ACKNOWLEDGMENT}

Part of this work was funded by the Department of Information Technology, MCIT, Government of India under the Visvesvarya Young Fellowship Scheme. L. Hanzo was supported by the ERC Advanced Fellow Grant Beam-me-up. 
TABLE II: Numerical example for $N=50, p_{m d}=0.2, p_{f a}=0.4, a=1, b=1, P_{p}=200$ and $P_{c}=100$.

\begin{tabular}{|l|l|l|l|l|l|l|}
\hline$\alpha$ & $\left(p_{p}, p_{c}\right)$ & $\left(\hat{p}_{m d}, \hat{p}_{f a}\right)$ & $\left(R_{p}, R_{s}\right)$ & $R_{p}+R_{s}$ & $\left(R_{p}, R_{s}\right) \mathrm{TS}$ & $\left(R_{p}+R_{s}\right) \mathrm{TS}$ \\
\hline$\downarrow$ & \multicolumn{5}{|c|}{$p_{o}=0.2$} \\
\hline 0.8 & $(0.25,0)$ & $(0,0.83)$ & $(1.008,0.61)$ & 1.62 & \\
0.2 & $(0.88,0.03)$ & $(0.024,0.42)$ & $(0.99,2.17)$ & 3.161 & $(0.85,2.26)$ & 3.106 \\
$\alpha^{*}=0.12$ & $(0.99,0.09)$ & $(0.08,0.4)$ & $(0.95,2.24)$ & 3.19 & & \\
\hline \multicolumn{7}{|c|}{$p_{o}=0.4$} \\
0.8 & $(0.5,0)$ & $(0,0.8)$ & $(2.07,0.55)$ & 2.62 & & \\
\hline 0.8 & $(0.99,0.25)$ & $(0.1,0.4)$ & $(1.91,1.7)$ & 3.61 & $(1.74,1.72)$ & 3.46 \\
$\alpha^{*}=0.3$ & $(0.99,0.15)$ & $(0.05,0.4)$ & $(1.99,1.69)$ & 3.68 & & \\
\hline
\end{tabular}

\section{REFERENCES}

[1] I. Mitola, J. and J. Maguire, G.Q., "Cognitive radio: making software radios more personal," IEEE Personal Communications, vol. 6, pp. 13 -18 , aug 1999.

[2] S. Haykin, "Cognitive radio: brain-empowered wireless communications," IEEE Journal on Selected Areas in Communications, vol. 23, pp. $201-220$, feb. 2005.

[3] Y.-C. Liang, K.-C. Chen, G. Li, and P. Mahonen, "Cognitive radio networking and communications: an overview," IEEE Transactions on Vehicular Technology, vol. 60, pp. 3386-3407, Sept 2011.

[4] A. Patel, M. Z. A. Khan, S. N. Merchant, U. B. Desai, and L. Hanzo, "The achievable rate of interweave cognitive radio in the face of sensing errors," IEEE Access, vol. 5, pp. 8579-8605, 2017.

[5] T. Yucek and H. Arslan, "A survey of spectrum sensing algorithms for cognitive radio applications," IEEE Communications Surveys Tutorials , vol. 11, pp. $116-130$, quarter 2009.

[6] I. F. Akyildiz, B. F. Lo, and R. Balakrishnan, "Cooperative spectrum sensing in cognitive radio networks: A survey," Phys. Commun., vol. 4, pp. 40-62, Mar. 2011.

[7] J. Lunden, V. Koivunen, and H. Poor, "Spectrum exploration and exploitation for cognitive radio: Recent advances," IEEE Signal Processing Magazine, vol. 32, pp. 123-140, May 2015.

[8] I. Christian, S. Moh, I. Chung, and J. Lee, "Spectrum mobility in cognitive radio networks," IEEE Communications Magazine, vol. 50, pp. 114-121, June 2012.

[9] L. Sboui, Z. Rezki, and M.-S. Alouini, "Achievable rate of spectrum sharing cognitive radio systems over fading channels at low-power regime," IEEE Transactions on Wireless Communications, vol. 13, pp. 6461-6473, Nov 2014.

[10] N. Devroye, P. Mitran, and V. Tarokh, "Achievable rates in cognitive radio channels," IEEE Transactions on Information Theory, vol. 52, pp. 1813 - 1827, may 2006.

[11] W. Liang, S. X. Ng, J. Feng, and L. Hanzo, "Pragmatic distributed algorithm for spectral access in cooperative cognitive radio networks," IEEE Transactions on Communications, vol. 62, pp. 1188-1200, April 2014.

[12] C. Li and H. Dai, "On the throughput scaling of cognitive radio ad hoc networks," in IEEE INFOCOM 2011 Proceedings, pp. 241-245, April 2011.

[13] W. El-Hajj, H. Safa, and M. Guizani, "Survey of security issues in cognitive radio networks," Journal of Internet Technology, vol. 12, pp. 181-198, March 2011.

[14] A. U. Rehman, C. Dong, L. L. Yang, and L. Hanzo, "Performance of cognitive stop-and-wait hybrid automatic repeat request in the face of imperfect sensing," IEEE Access, vol. 4, pp. 5489-5508, 2016.

[15] L. Hanzo, J. C. S. Cheung, R. Steele, and W. T. Webb, "A packet reservation multiple access assisted cordless telecommunication scheme," IEEE Transactions on Vehicular Technology, vol. 43, pp. 234-244, May 1994.
[16] S. C. Borst and D. Mitra, "Virtual partitioning for robust resource sharing: computational techniques for heterogeneous traffic," IEEE Journal on Selected Areas in Communications, vol. 16, no. 5, pp. 668678, Jun 1998. 
Aaqib Patel (S'13, M'18) received the B. Tech. degree in Electronics and Communications Engineering from NIT Warangal in 2010 and Ph.D from IIT Bombay in 2016. He is currently a DST INSPIRE faculty at IIT Hyderabad. Prior to joining IIT Hyderabad he was a research scientist at IIT Bombay. His research areas include Information Theory, Game Theory, Cyber Physical Systems and Markov Decision Theory with application in the Wireless Communication. His Ph.D. work is focused on Information Theoretic Models for Cognitive Radio under Practical Constraints.

Mohammed Zafar Ali Khan (SM'17) received the B.E degree in Electronics and communications from Osmania University, Hyderabad, India, the M.Tech degree in Electrical Engineering from IIT Delhi and Ph.D. degree in Electrical Communication Engineering from IISc,Bangalore, in 1996,in 1998 and 2003 respectively. He was a design engineer with Sasken, Bangalore in 1999, a senior design engineer with Silica semiconductors, Bangalore from 20032005, and a senior member of Technical staff at Hellosoft, India in 2005 and Assistant Professor at IIIT Hyderabad from 2006-2009. He is currently with IIT Hyderabad as an Associate professor. He has more than ten years of experience in teaching and research. He has made note worthy contributions to Space time codes. The Space time block codes designed by him have been adopted by the WiMAX Standard. He is also recipient of INAE young engineer award 2006. His research are in coded modulation, Space-Time Coding, and Signal Processing for Wireless Communications. He has been a chief investigator for a number of sponsored and consultancy projects. He is a reviewer for many international and national journals and conferences. He also author of book Single and Double Symbol Decodable Space time block codes from Lambert academic press, Germany.

S. N. Merchant received his B. Tech, M. Tech, and PhD degrees all from Department of Electrical Engineering, Indian Institute of Technology Bombay, India. Currently he is a Professor in Department of Electrical Engineering at IIT Bombay. He has more than 30 years of experience in teaching and research. Dr. Merchant has made significant contributions in the field of signal processing and its applications. His noteworthy contributions have been in solving state of the art signal and image processing problems faced by Indian defence. His broad area of research interests are wireless communications, wireless sensor networks, signal processing, multimedia communication, and image processing and has published extensively in these areas. He is a coauthor with his students who have won Best Paper Awards. He has served on Technical Program Committees of many IEEE premier conferences. He serves on the Editorial Board of two International Journals: International Journal of Distributed Sensor Networks, and International Journal of Ultra Wideband Communications and Systems. He has been a chief investigator for a number of sponsored and consultancy projects. He has served as a consultant to both private industries and defence organizations. He is on the Academic and Governing Advisory Boards of different engineering colleges in India. $\mathrm{He}$ is a Fellow of IETE. He is a recipient of 10th IETE Prof. S. V. C. Aiya Memorial Award for his contribution in the field of detection and tracking. $\mathrm{He}$ is also a recipient of 9th IETE SVC Aiya Memorial Award for Excellence in Telecom Education. He is a winner of the 2013 VASVIK Award in the category of Electrical \& Electronic Sciences \& Technology.
Uday B. Desai (S75-M78-SM96) received the B. Tech. degree from the Indian Institute of Technology (IIT) Kanpur, Kanpur, India, in 1974, the M.S. degree from the State University of New York, Buffalo, NY, USA, in 1976, and the Ph.D. degree from The Johns Hopkins University, Baltimore, MD, USA, in 1979, all in electrical engineering. Since June 2009, he has been the Director of IIT Hyderabad, India. From 1979 to 1984, he was an Assistant Professor with the School of Electrical Engineering and Computer Science Department, Washington State University, Pullman, WA, USA, and an Associate Professor with the same university from 1984 to 1987. From 1987 to May 2009, he was a Professor with the Electrical Engineering Department, IIT-Bombay, Mumbai, India. He was a Dean of Students with the IIT-Bombay from August 2000 to July 2002. He was a Visiting Associate Professor with Arizona State University, Purdue University and Stanford University. He was a Visiting Professor with EPFL, Lausanne, Switzerland, during the summer of 2002. From July 2002 to June 2004, he was the Director of the HPIITM R\& D Laboratory, IIT-Madras, Chennai, India. His research interests include wireless communication, wireless sensor networks, statistical signal processing, multimedia, image and video processing, artificial neural networks, computer vision, and wavelet analysis. Dr. Desai is a Fellow of the Indian National Science Academy (INSA) and the Indian National Academy of Engineering (INAE). He is one of the founding member of COMSNETS and also the Society for Cancer Research and Communication. He was the Chair for the IEEE Bombay Section 2006-2008. He was also on the Visitation Panel for the University of Ghana. He is on the Board of Tata Communications Limited. He was the recipient of the J. C. Bose Fellowship. He was also the recipient of the Excellence in Teaching Award from IIT-Bombay for 2007.

Lajos Hanzo (M'91-SM'92-F'04) received the D.Sc. degree in electronics in 1976 and the Ph.D. degree in 1983. During his 40-year career in telecommunications he has held various research and academic posts in Hungary, Germany, and the U.K. Since 1986, he has been with the School of Electronics and Computer Science, University of Southampton, U.K. He has successfully supervised $111 \mathrm{Ph}$.D. students. In 2016, he was admitted to the Hungarian Academy of Science. He is currently directing a 50-strong academic research team, where he is involved in a range of research projects in the field of wireless multimedia communications sponsored by industry, the Engineering and Physical Sciences Research Council, U.K., the European Research Councils Advanced Fellow Grant, and the Royal Societys Wolfson Research Merit Award. He is an enthusiastic supporter of industrial and academic liaison and he offers a range of industrial courses. He has co-authored $18 \mathrm{John}$ Wiley/IEEE Press books on mobile radio communications totaling in excess of 10000 pages, and published over 1681 research contributions on IEEE Xplore. He was FREng, FIET, and a fellow of the EURASIP. In 2009, he received an honorary doctorate from the Technical University of Budapest and in 2015 from the University of Edinburgh. He has served as the TPC chair and the general chair of various IEEE conferences, presented keynote lectures and has received a number of distinctions. He is also a Governor of the IEEE VTS. From 2008 to 2012, he was Editor-in-Chief of the IEEE Press and a Chaired Professor with Tsinghua University, Beijing. He is the Chair in telecommunications with the University of Southampton. He has 33,000+ citations and an h-index of 73 . 